ARTICLE

\title{
Norovirus infection causes acute self-resolving diarrhea in wild-type neonatal mice
}

\author{
Alexa N. Roth ${ }^{1,5}$, Emily W. Helm¹,5, Carmen Mirabelli (1) ${ }^{2}$, Erin Kirsche ${ }^{1}$, Jonathan C. Smith ${ }^{1}$, Laura B. Eurell ${ }^{3}$, \\ Sourish Ghosh ${ }^{4}$, Nihal Altan-Bonnet ${ }^{4}$, Christiane E. Wobus (ib ${ }^{2} \&$ Stephanie M. Karst (iD) ${ }^{1 凶}$
}

Human noroviruses are the leading cause of severe childhood diarrhea worldwide, yet we know little about their pathogenic mechanisms. Murine noroviruses cause diarrhea in interferon-deficient adult mice but these hosts also develop systemic pathology and lethality, reducing confidence in the translatability of findings to human norovirus disease. Herein we report that a murine norovirus causes self-resolving diarrhea in the absence of systemic disease in wild-type neonatal mice, thus mirroring the key features of human norovirus disease and representing a norovirus small animal disease model in wild-type mice. Intriguingly, lymphocytes are critical for controlling acute norovirus replication while simultaneously contributing to disease severity, likely reflecting their dual role as targets of viral infection and key components of the host response.

\footnotetext{
${ }^{1}$ Department of Molecular Genetics \& Microbiology, College of Medicine, University of Florida, Gainesville, FL, USA. ${ }^{2}$ Department of Microbiology and Immunology, University of Michigan, Ann Arbor, MI, USA. ${ }^{3}$ Office of Research, College of Medicine, University of Florida, Gainesville, FL, USA. ${ }^{4}$ Laboratory of Host-Pathogen Dynamics, National Heart Lung and Blood Institute, National Institutes of Health, Bethesda, MD, USA. ${ }^{5}$ These authors contributed equally: Alexa N. Roth, Emily W. Helm. ${ }^{\bowtie}$ email: skarst@ufl.edu
} 
H uman noroviruses are the leading cause of severe childhood diarrhea and gastroenteritis outbreaks worldwide ${ }^{1-3}$ yet we know very little about their pathogenic mechanisms. While human noroviruses cause modest diarrhea in gnotobiotic piglets, gnotobiotic calves, and miniature piglets ${ }^{4-6}$, a limiting factor in studying norovirus pathogenesis is the lack of tractable small animal models that recapitulate key features of disease observed in infected individuals. The first murine norovirus, MNV-1, was discovered nearly two decades $\mathrm{ago}^{7}$ and many other MNV strains have been reported since then. MNV strains segregate into two phenotypic categories which differ in many aspects of pathogenesis, including their rate of clearance from infected hosts and cell tropism. MNV-1 is the prototype acute strain. It infects immune cells in the gut-associated lymphoid tissue (GALT) and reaches peak intestinal titers 1-2 days post-infection (dpi) with clearance from the host by $7-14 \mathrm{dpi}^{8}$. MNV-3 and MNV-CR6 are commonly referred to as persistent strains since they establish a life-long colonic infection ${ }^{9-11}$. The persistent reservoir is a rare type of intestinal epithelial cell, the tuft cell ${ }^{12}$. While major strides have been made in understanding the cellular and tissue tropism of noroviruses using the MNV model system, a limitation of the murine model is the lack of diarrhea in wild-type laboratory mice infected with any of the MNV strains studied to date ${ }^{13-15}$. While MNV-1 does not cause overt disease in adult wild-type mice, infection of interferon (IFN)-deficient Stat $1^{-/-}$and IFN $\alpha \beta \gamma R^{-/-}$adult mice causes severe weight loss and diarrhea similar to human norovirusinfected individuals ${ }^{13-16}$. The persistent strains $\mathrm{MNV}-3$ and MNV-CR6 cause less disease than MNV-1 in IFN-deficient mouse strains ${ }^{14,17}$.

Like noroviruses, rotaviruses cause severe acute diarrhea in people yet fail to cause disease in adult wild-type mice. However, it is well-established that neonatal wild-type mice are susceptible to rotavirus-induced diarrhea ${ }^{18-20}$. Moreover, disease severity following human norovirus infection is greater in younger children $^{21-23}$. Thus, we tested whether MNV causes disease in genetically wild-type neonatal mice. Herein, we report that MNV1 causes diarrhea in neonatal BALB/c mice while MNV-3 and MNV-CR6 cause attenuated disease in this model. In addition, we find that lymphocytes both contribute to disease severity and are critical for controlling acute replication which likely reflects their dual role as both cellular targets and components of the host immune response.

\section{Results}

Development of a neonatal mouse model. We first confirmed the established virulence profile of MNV strains in IFN-deficient adult mice by infecting adult wild-type or Ifnar1-/- mice with MNV-1, MNV-3, or MNV-CR6. While MNV-1 caused weight loss and fatality of Ifnar $1^{-1-}$ mice by $4 \mathrm{dpi}, \mathrm{MNV}-3$ and MNV-CR6 failed to cause overt disease (Fig. 1a, b). Overall, these results indicate that $\mathrm{MNV}$ can cause disease in adult mice but virulence is regulated by an IFN response and viral genetic determinants. Moreover, MNV-1 infection of IFN-deficient mice causes systemic disease and mortality in addition to diarrhea which are not hallmarks of human norovirus infection. Thus, we sought to develop a more relevant disease model by testing whether genetically wildtype neonatal mice develop self-resolving diarrhea.

Indeed, oral inoculation of 3-day-old $\mathrm{BALB} / \mathrm{c}$ mice with acute MNV-1 caused diarrhea beginning at $42 \mathrm{~h}$ post-infection (hpi) and resolving by $4 \mathrm{dpi}$ (Fig. 1c, d, and Supplementary Fig. 1c), with a $91 \%$ incidence (Fig. 1e). Virus replication was required for diarrhea induction, as demonstrated by the absence of disease in pups inoculated with UV-inactivated MNV-1 (Fig. 1d, e and Supplementary Fig. 1b). Furthermore, MNV-1 diarrhea occurred in a dose-dependent manner (Fig. 2a, b). The incidence of diarrhea was significantly higher in MNV-1-infected 3-day-old $\mathrm{BALB} / \mathrm{c}$ mice compared with 4 -day-old $\mathrm{BALB} / \mathrm{c}$ mice, with a $91 \%$ versus $49 \%$ incidence, respectively (Fig. 2c, d), and slightly, although not significantly, higher in female BALB/c mice than in male mice (Fig. 2e, f). MNV-1 induced diarrhea in BALB/c mice at two independent research institutions (University of Florida and the National Institutes of Health). For all other experiments described herein, we used 3-day-old BALB/c mice of mixed sex. We next tested whether the persistent strains MNV-3 and MNVCR6 likewise caused diarrhea in neonatal mice. Compared with the $91 \%$ incidence of diarrhea in MNV-1-infected BALB/c pups, MNV-3 and MNV-CR6 caused diarrhea in $43 \%$ and $32 \%$ of pups, respectively (Fig. 1d, e and Supplementary Fig. 1d, e). Collectively, these data reveal that MNV-1 infection causes selfresolving acute diarrhea in neonatal $\mathrm{BALB} / \mathrm{c}$ mice and that $\mathrm{MNV}$ 3 and MNV-CR6 cause a reduced incidence of diarrhea relative to MNV-1, mirroring their virulence patterns previously observed in adult IFN-deficient mice ${ }^{14,17}$.

MNV infection induces pathological changes in neonatal mice. We next determined whether MNV-induced diarrhea is associated with histopathological changes along the intestinal tract. A number of human volunteer studies have been carried out where intestinal biopsies were obtained from subjects at the time of norovirus symptoms ${ }^{24-29}$. While the epithelium remained intact with no gross lesions and there was only a minimal to moderate increase in lamina propria cellularity noted in these patients, there were consistent infection-associated pathologies including villous blunting and broadening, vacuolated and disorganized epithelial cells, and crypt cell hyperplasia. Histopathological changes in MNV-infected neonatal mice are entirely consistent with these human biopsies: While the epithelium itself remained intact and only modest inflammation was observed, there was villous broadening, epithelial disorganization and vacuolization, and crypt cell hyperplasia (Fig. 3a and Supplementary Fig. 2). We also observed lacteal dilation, a pathology previously noted in MNV-4-infected Stat $1^{-1-}$ mice ${ }^{30}$ that can be indicative of impaired fat absorption. Overall pathology was more pronounced in the proximal half of the small intestine than the distal half, and it positively correlated with diarrhea incidence when comparing intestinal sections from pups infected with MNV-1, MNV-3, and MNV-CR6 (Fig. 3b, c and Supplementary Fig. 2).

MNV-1 replicates in subepithelial cells of the small intestine. Because MNV-1 caused the highest incidence of diarrhea in neonates, we further characterized its pathogenesis in this model. Virus titers were similar in all regions of the gastrointestinal tract at the peak of disease, $2 \mathrm{dpi}$, and splenic titers were high at this time point (Fig. 4a). In order to confirm viral replication in vivo, we infected neonatal mice with light-sensitive neutral red-labeled MNV-1 which enables differentiation between input and newly synthesized virus ${ }^{31,32}$. Newly synthesized virus was detectable in all tissues as early as $0.5 \mathrm{dpi}$ and peaked at $1 \mathrm{dpi}$ (Fig. 4b). We next examined the cell tropism of MNV-1 during symptomatic infection. We previously showed that MNV-1 infects immune cells in the GALT of the distal small intestine of wild-type adult mice $^{8}$. To determine whether there is a similar tropism during symptomatic norovirus infection, intestinal sections from MNV1 -infected neonates were hybridized with probes to the viral positive- and negative-sense RNA species and analyzed by RNAscope-based in situ hybridization. At $1 \mathrm{dpi}$, the peak of viral titers, viral positive-sense RNA was detected in both epithelial and subepithelial cells in the intestinal villi and GALT but negative-sense RNA indicative of viral replication was exclusively 
a

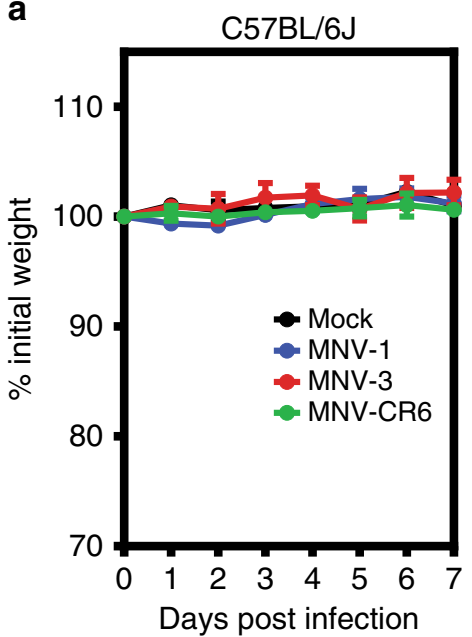

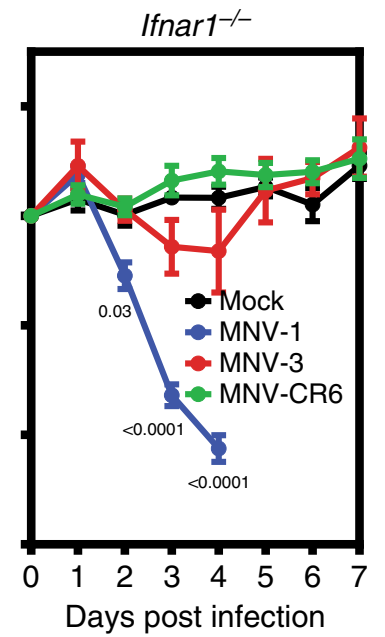

b

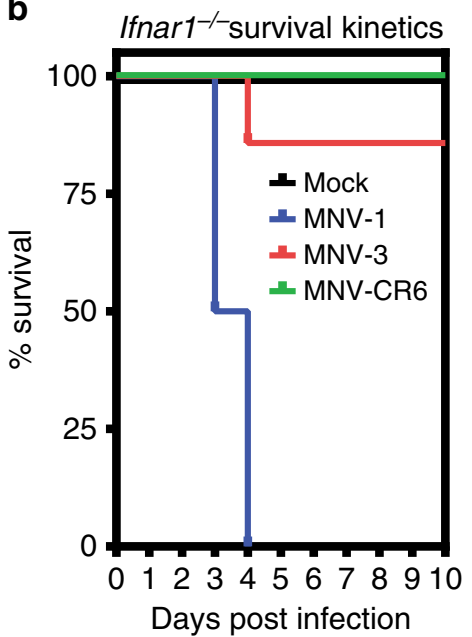

c

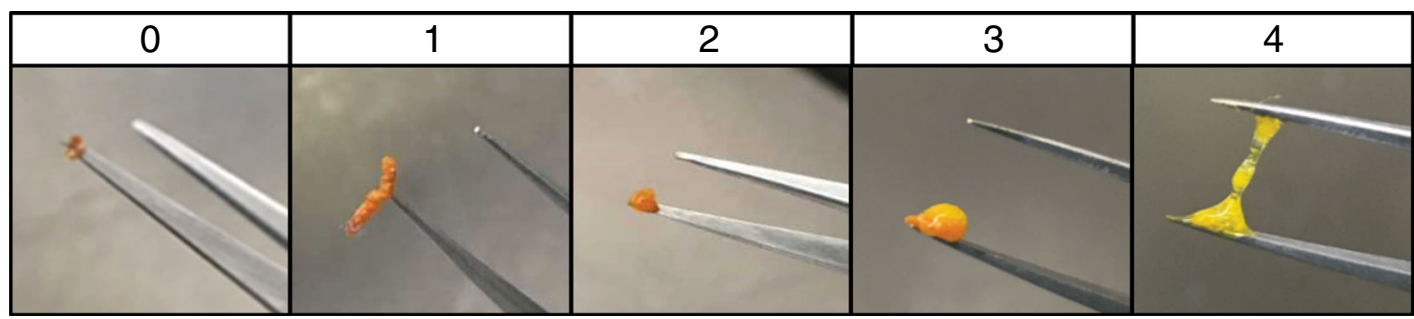

d

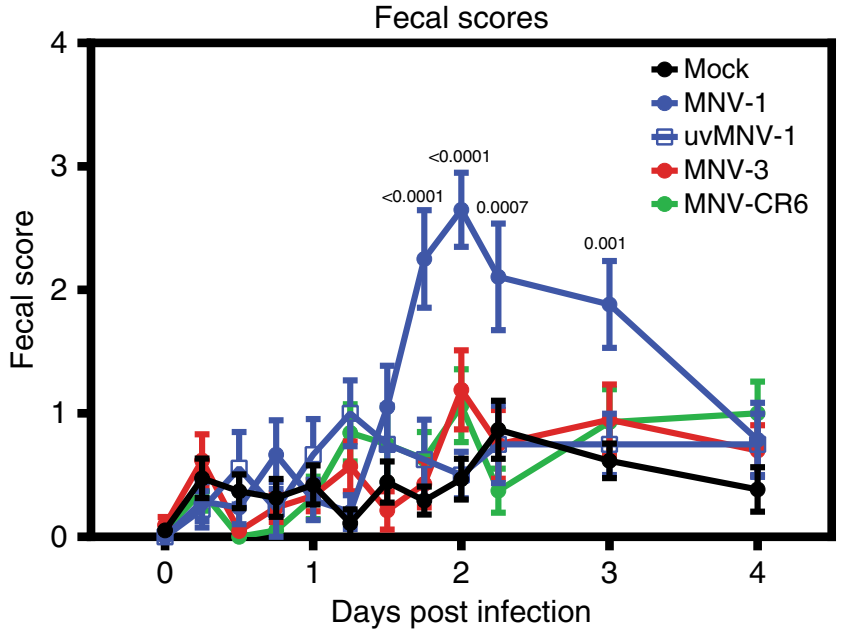

e

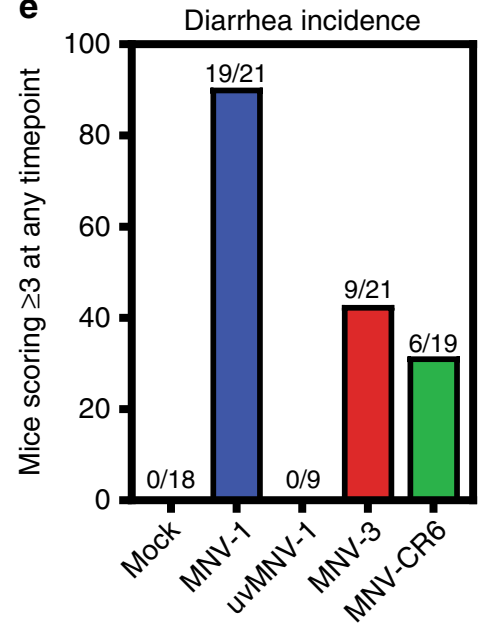

Fig. 1 MNV infection causes disease in a virus strain-dependent manner. a, b Groups of C $57 \mathrm{BL} / 6 \mathrm{~J}(n=6$ for $\mathrm{mock}, n=8$ for $M N V-1, n=6$ for $M N V-3$,

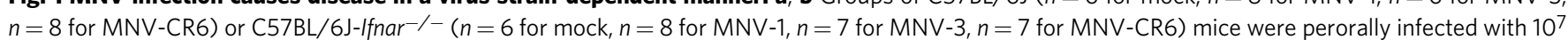
TCID 50 units of MNV-1 (blue), MNV-3 (red), MNV-CR6 (green), or mock (black) inoculum and followed for weight loss (a) and survival (b). c Representative images of fecal samples indicating each score used to assess for diarrhea are shown. d, e Groups of 3-day-old BALB/c pups were infected with $10^{8} \mathrm{TCID}_{50}$ units of MNV-1 (blue), UV-inactivated MNV-1 (white with blue border), MNV-3 (red), MNV-CR6 (green), or mock (black) inoculum by oral gavage. Pups ( $n=19$ for mock, $n=21$ for MNV-1, $n=9$ for UV-inactivated MNV-1, $n=21$ for MNV-3, $n=19$ for MNV-CR6) were monitored for fecal consistency by palpating their abdomens (d). The proportion of mice scoring a 3 or 4 at any time point over the 5 days course of infection is presented as the incidence of diarrhea (e). Error bars denote standard errors of mean in all figures. $P$ values were determined using two-way ANOVA with corrections for multiple comparisons. Source data are provided as a Source data file.

detected in subepithelial cells in the GALT and the lamina propria (Fig. 5a, b). The presence of viral positive-sense, but not negative-sense, viral RNA in intestinal epithelial cells suggests that either virions can enter these cells but not replicate; or that replication occurs in these cells at a level below the limit of detection of our assay. Substantial viral replication was detected in splenocytes, consistent with high splenic titers and with a predominantly immune cell tropism. At $2 \mathrm{dpi}$, the peak of diarrhea, there was still viral positive-sense RNA along the gastrointestinal tract but minimal negative-sense viral RNA, demonstrating that viral replication in the gut has primarily been controlled by this time point (Fig. $5 \mathrm{c}-\mathrm{e}$ ). The pattern of viral positive-sense RNA at this time point was variable among the seven pups analyzed: Four pups contained positive-sense RNA primarily in subepithelial cells of the GALT and lamina propria similar to $1 \mathrm{dpi}$ (Fig. 5c) whereas two pups contained 
a

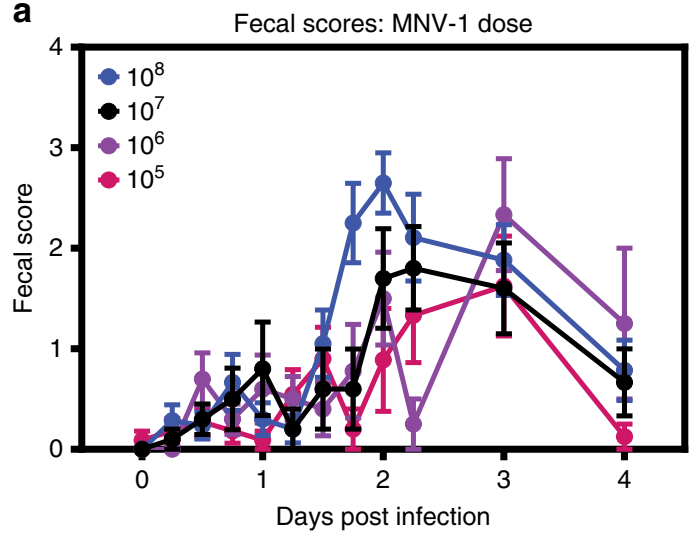

C

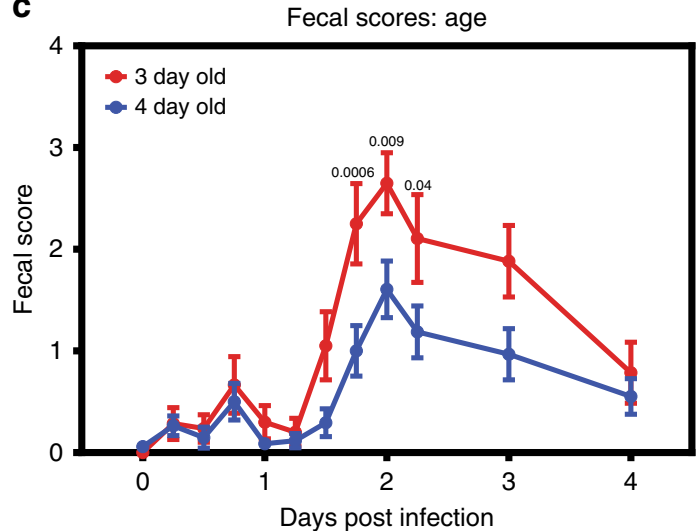

e

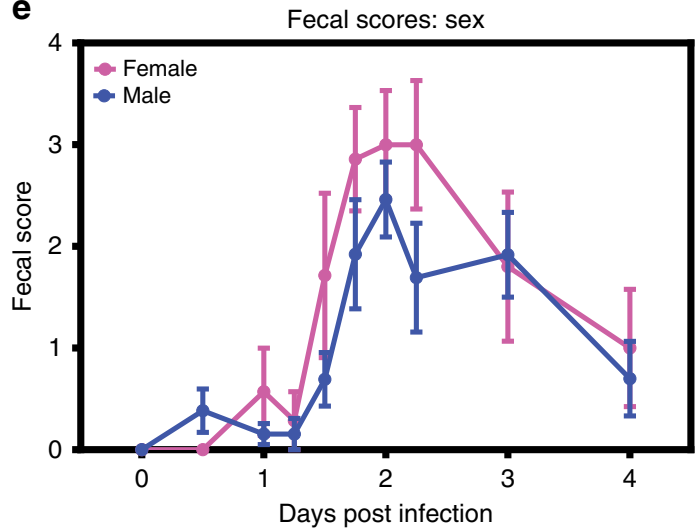

b

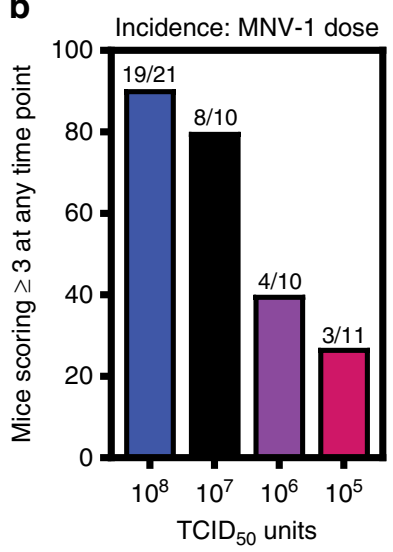

d

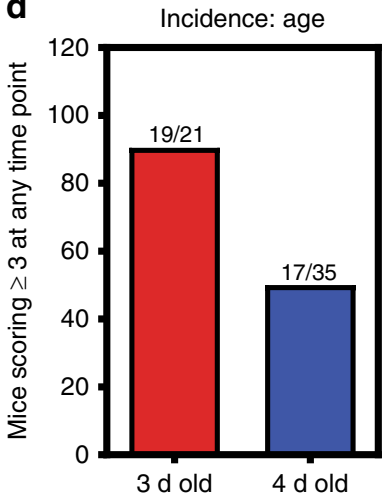

f

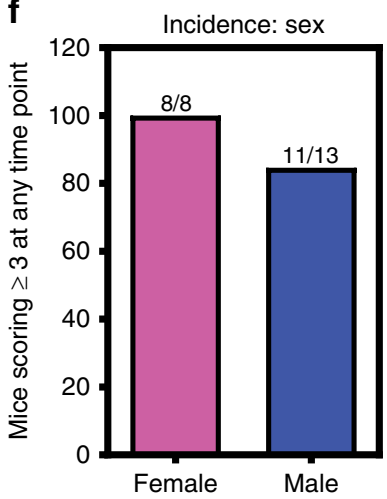

Fig. 2 Dose, host age, and sex influence MNV disease severity in neonatal mice. a Groups of 3-day-old BALB/c pups were infected with $10^{8}$ ( $n=21$, blue), $10^{7}$ ( $n=10$, black), $10^{6}$ ( $n=10$, purple), or $10^{5}(n=11$, pink) TCID 50 units of MNV-1 by oral gavage and monitored for fecal consistency by palpating their abdomens. $\mathbf{b}$ The proportion of mice scoring a 3 or 4 at any time point over the 5 days course of infection is presented as the incidence of diarrhea. c, $\mathbf{d}$ Data stratified by age from groups of 3- and 4-day-old BALB/c pups ( $n=21$ for 3-day old (red); $n=35$ for 4-day old (blue)) infected with $10^{8}$ TCID ${ }_{50}$ units of MNV-1 by oral gavage and monitored for fecal inconsistency. Data presented as fecal scores over time (c) and incidence (d). e, $\mathbf{f}$ Data stratified by $\operatorname{sex}\left(n=8\right.$ for female (pink); $n=13$ for male (blue)) from groups of 3-day-old BALB/c pups infected with $10^{8} \mathrm{TCID}_{50}$ units of MNV-1 by oral gavage and monitored for fecal inconsistency. Data presented as fecal scores over time (e) and incidence (f). Error bars denote standard errors of mean in all figures. $P$ values were determined using two-way ANOVA with corrections for multiple comparisons. Source data are provided as a Source data file.

positive-sense viral RNA primarily in villous epithelial cells (Fig. 5d). Intriguingly, this signal was confined to the distal region of the small intestine and to the upper half of the villi in this region. The remaining pup displayed an intermediate phenotype, with positive-sense RNA detected in subepithelial and epithelial cells. All pups had substantial positive-sense RNA and variable amounts of negative-sense RNA in the spleen at 2 dpi. Overall, viral replication was observed in subepithelial cells in the intestine and spleen.
Lymphocytes exhibit dual roles during acute MNV-1 infection. To begin dissecting the role of specific cellular targets in norovirus disease, we infected lymphocyte-deficient Rag1 $^{-/-}$neonates with MNV-1. Surprisingly, virus titers at $2 \mathrm{dpi}$ were significantly higher in $R a g 1^{-1-}$ mice compared with wild-type controls (Fig. 6a). In spite of these increased virus titers, the incidence of diarrhea was reduced in $\mathrm{Rag1}^{-/}$mice $(56 \%)$ compared with wild-type mice (90\%) (Fig. 6b, c). These collective results suggest that lymphocytes play opposing roles during acute 
a
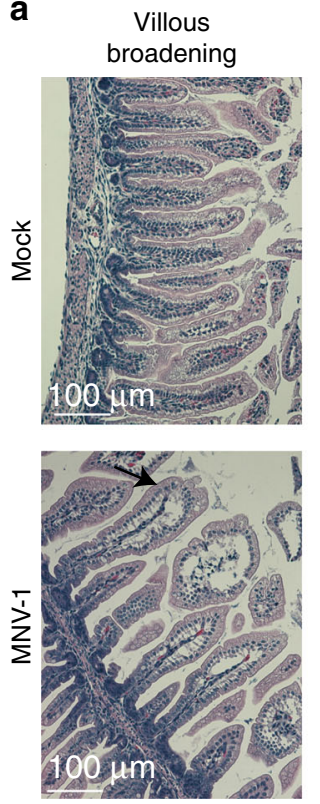

Epithelial cell vacoulization
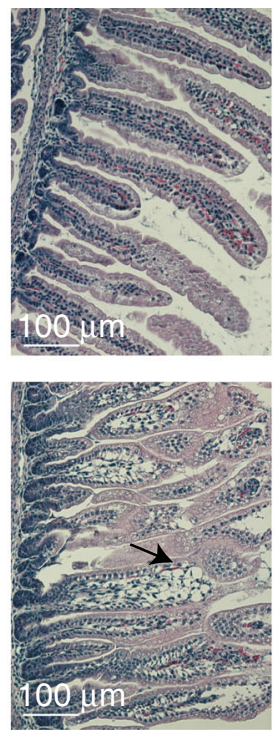

b

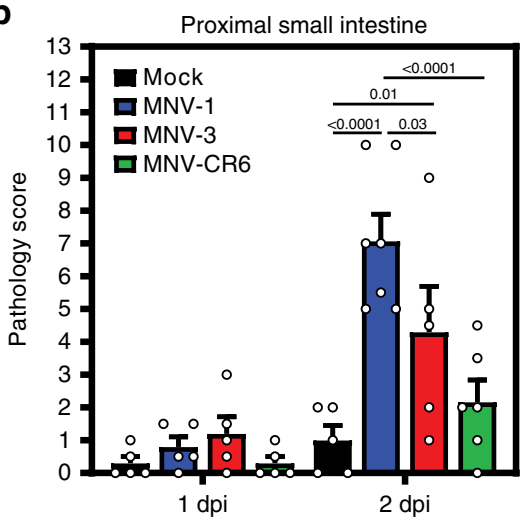

Lacteal
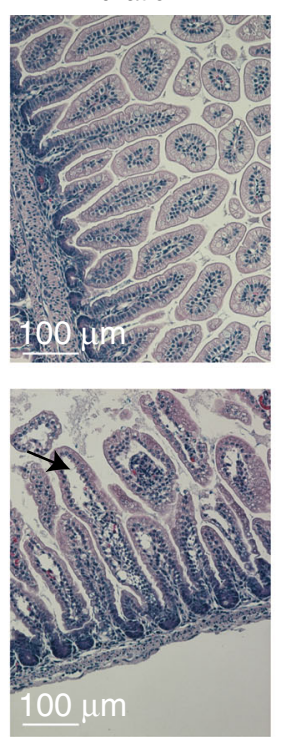

Crypt hyperplasia
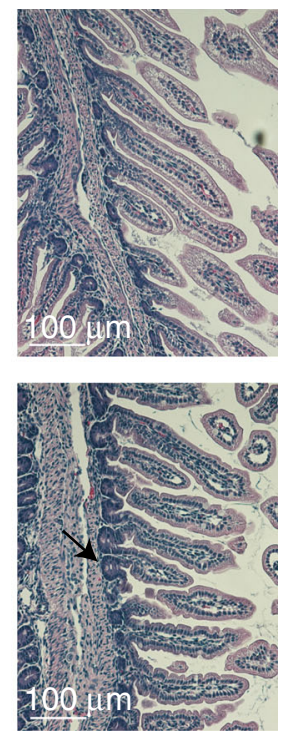

C

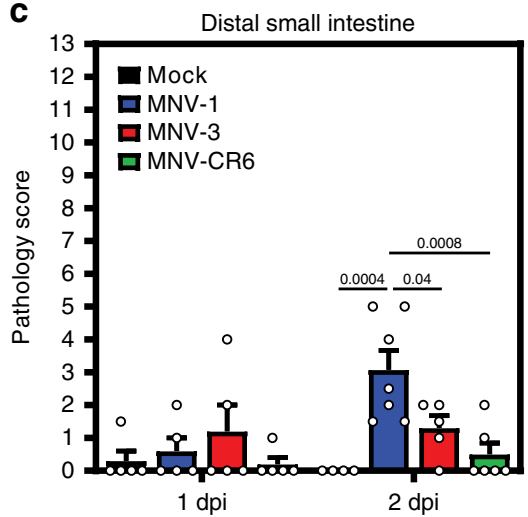

Fig. 3 Neonatal mice infected with MNV-1 show pathological changes. Small intestinal sections collected from neonates infected with $10^{8} \mathrm{TCID}_{50}$ units of MNV-1 (blue), MNV-3 (red), MNV-CR6 (green), or mock (black) inoculum at 1 dpi ( $n=5$ for mock, $n=5$ for MNV-1, $n=5$ for MNV-3, $n=5$ for MNVCR6) and $2 \mathrm{dpi}$ ( $n=5$ for mock, $n=7$ for MNV-1, $n=5$ for MNV-3, $n=6$ for MNV-CR6) were stained with hematoxylin and eosin. Representative images of notable pathology are shown (a). Sections were scored blindly by an animal veterinarian for pathological changes in the proximal small intestine (b) and distal small intestine (c). Scale bars denote $100 \mu \mathrm{m}$. Error bars denote standard errors of mean in all figures. $P$ values were determined using two-way ANOVA with corrections for multiple comparisons. Source data are provided as a Source data file.
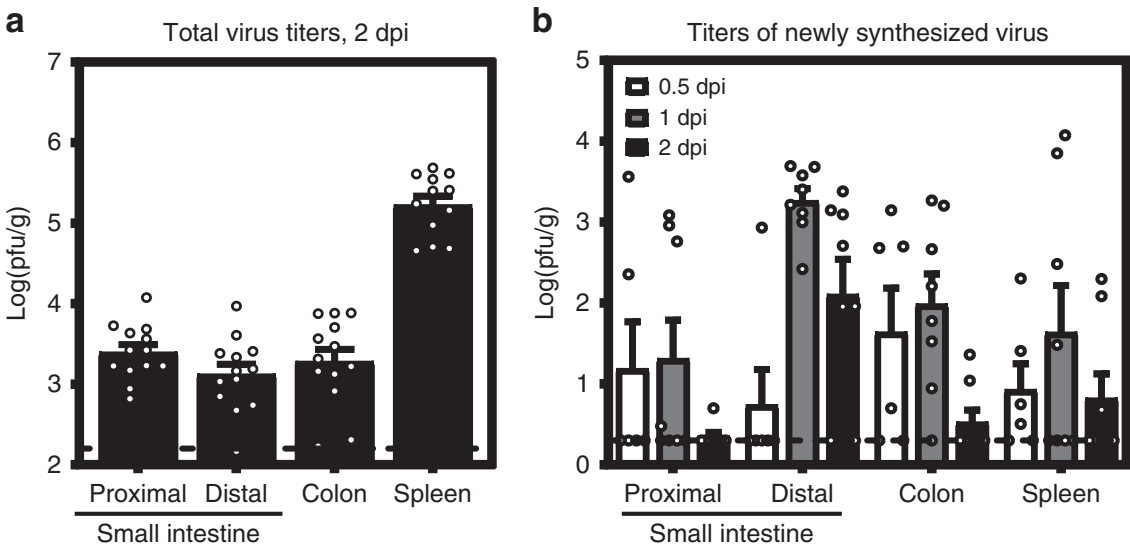

Fig. 4 MNV-1 establishes productive infection in neonatal mice. a Three-day-old BALB/c pups $(n=13)$ were infected with $10^{8}$ TCID 50 units of MNV-1. At $2 \mathrm{dpi}$, viral titers were determined in the indicated segments of the intestinal tract and spleen by plaque assay. $\mathbf{b}$ Three-day-old BALB/c pups were infected with $2.5 \times 10^{4}$ pfu of neutral red-labeled MNV- 1 by oral gavage At $0.5 \mathrm{dpi}(n=5$, white), $1 \mathrm{dpi}(n=8$, gray), and $2 \mathrm{dpi}(n=8$, black), plaque assays were used to measure newly synthesized virus. Error bars denote standard errors of mean in all figures. Source data are provided as a Source data file. 
a

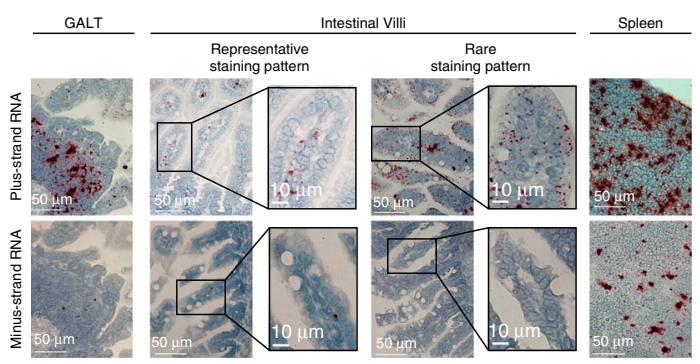

b

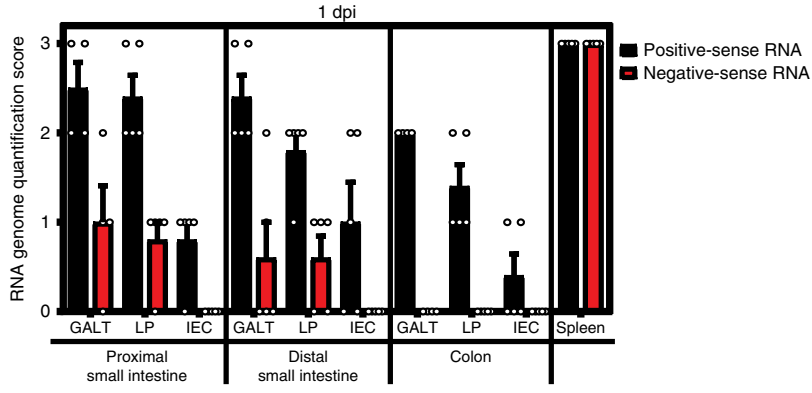

C

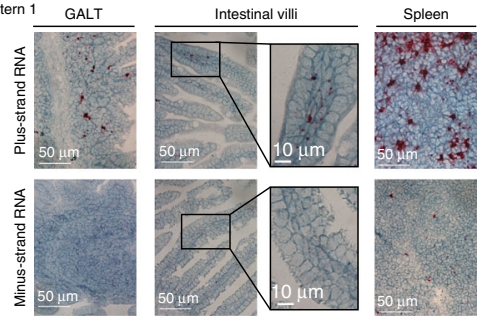

d

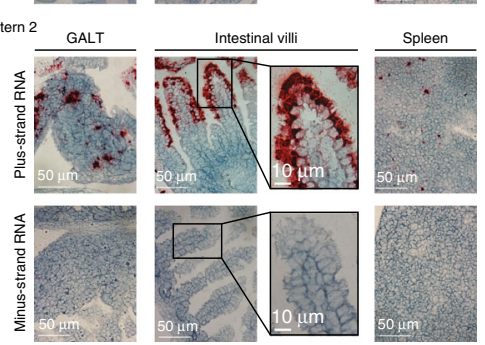

e

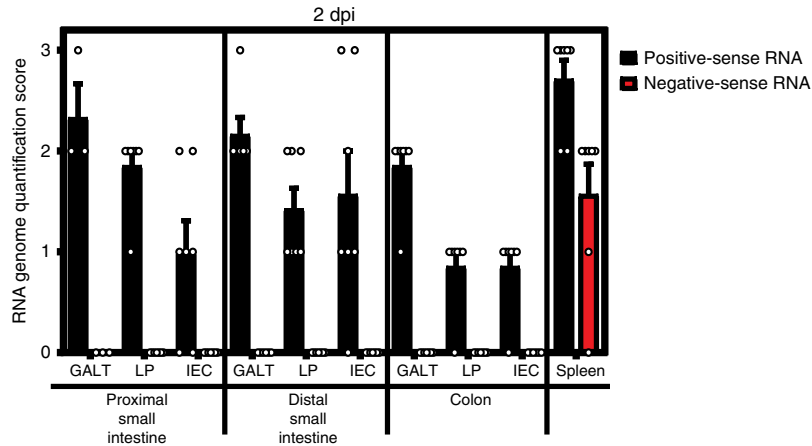

Fig. 5 MNV-1 replicates in intestinal subepithelial cells and splenocytes. Tissue sections from mock-inoculated or MNV-1-infected mice collected at $1 \mathrm{dpi}$ (a, b; $n=5$ per group) and $2 \mathrm{dpi}$ (c-e; $n=7$ per group) were probed for viral positive-sense and negative-sense RNA. Representative images of gut-associated lymphoid tissue (GALT), intestinal villi, and splenic tissue are shown for $1 \mathrm{dpi}$ (a) and at $2 \mathrm{dpi}$ for 2 distinct patterns (c, d). The amount of positive-sense (black) and negative-sense (red) viral RNA detected was scored for each pup, as described in the "Methods" at $1 \mathrm{dpi}$ (b) and $2 \mathrm{dpi}$ (e). Scale bars represent $50 \mu \mathrm{m}$ for all images except for insets where they represent $10 \mu \mathrm{m}$. Error bars denote standard errors of mean in all figures. Source data are provided as a Source data file. norovirus infection, being important for control of $\mathrm{MNV}-1$ replication while also contributing to disease severity possibly related to their permissiveness to the virus.

\section{Discussion}

The establishment of a small animal model to study norovirusinduced diarrhea in a genetically wild-type host that recapitulates key features of human disease will revolutionize the field's ability to understand the precise mechanisms underlying norovirus virulence and to rationally design antiviral therapies. Strengths of this neonatal mouse model compared with adult IFN-deficient mice are that disease is limited to the intestinal tract and selfresolving. Disease severity in this model is (i) regulated by viral genetics, (ii) age-dependent, (iii) not associated with disruption of the intestinal epithelium or notable inflammation, and (iv) associated with immune cell infection. In particular, the finding that highly genetically related MNV strains display differences in diarrhea severity will facilitate identification of viral virulence determinants. Underscoring the potential of this neonatal mouse model system, the discovery that neonatal mice are susceptible to rotavirus diarrhea was key to defining viral disease mechanisms such as the roles of rotavirus NSP4 as a viral enterotoxin ${ }^{33}$ and rotavirus stimulation of the enteric nervous system leading to increased intestinal transit associated with diarrhea ${ }^{34,35}$. The model system described herein holds a similar promise to reveal additional insights into norovirus disease mechanisms.

\section{Methods}

Cells and viruses. Cell lines used in this study tested negative for mycoplasma contamination and were not authenticated. The RAW 264.7 cell line (ATCC) was maintained in Dulbecco's modified Eagle medium (DMEM; Fisher Scientific) supplemented with $10 \%$ fetal bovine serum (FBS, Atlanta Biologicals), $100 \mathrm{U}$ penicillin $/ \mathrm{mL}$, and $100 \mu \mathrm{g} / \mathrm{mL}$ streptomycin. Stocks of recombinant MNV-1.CW3 (GenBank accession number KC782764, referred to as MNV-1), MNV-3 (GenBank accession number KC792553), and MNV-CR6 (GenBank accession number JQ237823.1) were generated ${ }^{36}$. 293T cells were seeded at $10^{6}$ cells per well in a 6well culture plate. The next day, $10^{6} 293 \mathrm{~T}$ cells were transfected with $5 \mu \mathrm{g}$ infectious clone using Lipofectamine 2000 (Life Technologies), cells were lysed by freeze-thaw at $-80^{\circ} \mathrm{C}$ after 1 day, and lysates titrated with a standard TCID $_{50}$ assay ${ }^{11}$ as described below and applied to RAW 264.7 cells. RAW 264.7 lysates were freezethawed when cultures displayed $90 \%$ cytopathic effect and supernatants were clarified by low-speed centrifugation followed by purification through a $25 \%$ sucrose cushion. The viral genomes of stocks were sequenced completely to confirm no mutations arose during stock generation and titered by a standard TCID assay $^{11}$ For the $\mathrm{TCID}_{50}$ assay, eight replicates each of multiple dilutions per sample were applied to RAW 264.7 cells and cytopathicity scored at $7 \mathrm{dpi}$. Three independent experiments were averaged to determine stock titers. A mock inoculum stock was prepared using RAW 264.7 lysate from uninfected cultures. A neutral red-labeled MNV-1 stock was generated by infecting RAW 264.7 cells at a MOI 0.05 with MNV-1 in the presence of $10 \mu \mathrm{g} / \mathrm{ml}$ neutral red (Sigma), a light-sensitive dye $\mathrm{e}^{31,37}$ After 2 days, supernatant was collected in a darkened room using a red photolight (Premier OMNI). Stocks were freeze-thawed twice and stored in a light safe box at $-80^{\circ} \mathrm{C}$. Viral titer was determined by neutral red virus plaque assay without white light exposure. For UV inactivation of MNV-1, MNV-1 was exposed to 250,000 $\mu \mathrm{J}$ $\mathrm{cm}^{-2} \mathrm{UV}$ for $30 \mathrm{~min}$. UV inactivation was confirmed using TCID 50 assay.

Mice. Specific-pathogen-free (SPF) mice used in this study were bred and housed in animal facilities at the University of Florida, National Institutes of Health, and University of Michigan. All animal experiments were performed in strict accordance with federal and university guidelines. The animal protocols were approved by the Institutional Animal Care and Use Committee at the University of Florida (study number 20190632), National Institutes of Health (study number H-0299), and University of Michigan (study number 00006658). The conditions in animal rooms used in this study fall within the standards set by the "Guide for the Care and Use of Laboratory Animals". Specifically, the average temperature is $72{ }^{\circ} \mathrm{F}$ and always is within $68-79^{\circ} \mathrm{F}$. Humidity ranges from 30 to $70 \%$. The room light cycle is 12-h on and 12-h off. Experiments were not performed in a blinded fashion nor was randomization used. Sample sizes were determined using power calculations based on our prior experience with the MNV system. Age- and sex-matched adult C57BL/6J (Jackson no. 000664) and C57BL/6J-Ifnar1 ${ }^{-/-}$(Jackson no. 010830) mice were used in adult mouse infections. Litters of 3- or 4-day-old BALB/c (Charles River no. 028) and 3-day-old BALB/c-Rag1-/- (Jackson no. 003145) pups including males and females were used in neonatal mouse infections. At least two independent litters were infected per condition in each experiment. 
a

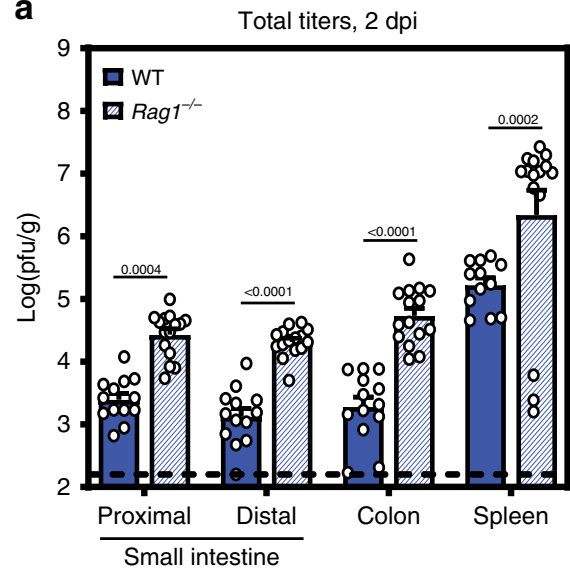

b

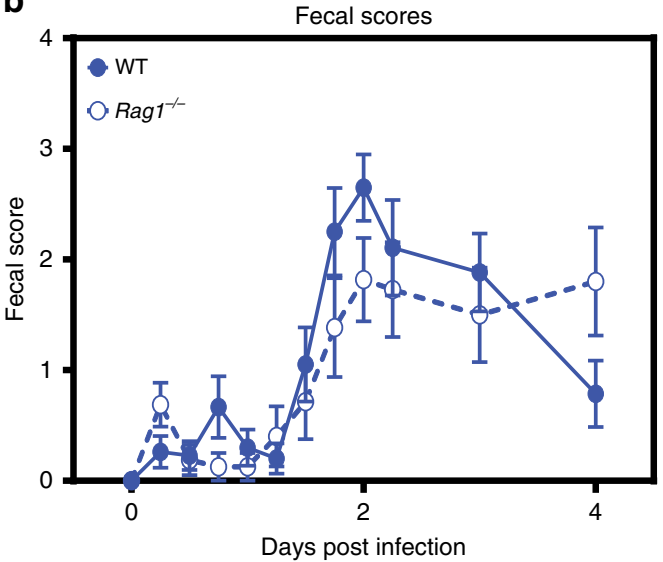

C

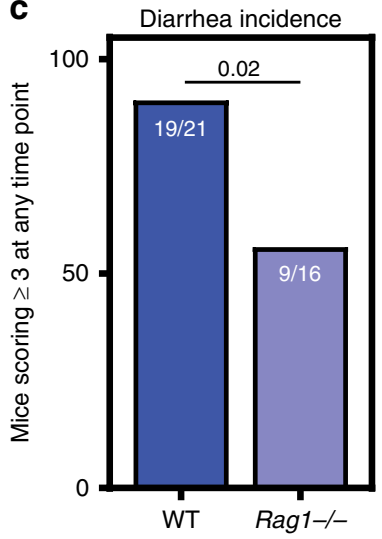

Fig. 6 MNV-1 infected Rag1 ${ }^{-/-}$pups show decreased diarrhea but increased viral titers. a Groups of 3-day-old BALB/c-Rag1-/- pups (blue stripes) were infected with $10^{8} \mathrm{TCID}_{50}$ units MNV-1 by oral gavage. At $2 \mathrm{dpi}(n=15)$, total viral titers were determined in the indicated segments of the intestinal tract and spleen by plaque assay, and compared with those of wild-type BALB/c pups (solid blue). b, c Additional BALB/c-Rag1-/- pups ( $n=16$, open circles) were infected with $10^{8} \mathrm{TCID}_{50}$ units MNV-1 by oral gavage and monitored for fecal consistency over a 5-day time course by palpating their abdomens. Fecal scores (b) and diarrhea incidence (c) were compared with those in wild-type BALB/c pups (solid blue). Error bars denote standard errors of mean in all figures. $P$ values were determined using two-way ANOVA with corrections for multiple comparisons for viral titers. For incidence, $P$ values were determined using a Mantel-Cox test. Source data are provided as a Source data file.

Mouse infections and other in vivo procedures. For all adult MNV infection experiments, 6- to 12-week-old, sex-matched mice were inoculated perorally (p.o.) with $10^{7} \mathrm{TCID}_{50}$ units of the indicated virus strain. For virulence assays in adult mice, C57BL/6J and C57BL/6J-Ifnar $1^{-/-}$mice were observed for weight loss and survival daily for up to 14 days. The daily weights were compared with day 0 weights to calculate the relative weight loss. For all neonatal mouse experiments, BALB/c or $\mathrm{BALB} / \mathrm{c}-\mathrm{Rag1} 1^{-/}$mice were infected at 3 or 4 days of age with the indicated dose of the indicated virus strain in a volume of $40 \mu \mathrm{l}$ delivered by oral gavage using a 22ga plastic feeding tube. Mice were observed every $6 \mathrm{~h}$ for the first $54 \mathrm{~h}$ post-infection (hpi), then daily thereafter until 4 dpi. Approximately $25 \%$ of pups failed to survive to the end of the experiment. A comparable frequency of lethality was observed in mock-inoculated and MNV-infected groups (see Source data file) so we presume that it resulted from stress associated with handling of the pups during the inoculation procedure either on the pup itself or the dam. This is not unexpected when handling pups as young as 3 days of age. At each time point, the abdomen of each mouse was palpated to induce defecation. Fecal condition was assessed based on color and consistency according to a 5-point scale: 0 , no defecation or solid; 1 , firm, orange, does not smear; 2 , pasty, orange or mixed color, does not smear; 3 , orange or yellow, semiliquid and smears; 4, yellow, liquid, and smears. For the purpose of calculating the incidence of diarrhea, any pup that received a score of 3 or 4 at any time point was considered positive for disease. For MNV titer determination, tissue samples were harvested and titrated by plaque assay ${ }^{14,36}$. For the plaque assays, tissues were weighed and homogenized by bead beating using $1.0 \mathrm{~mm}$ zirconia/silica beads. Dilutions of tissue samples were prepared and applied to RAW 264.7 cells. Plates were incubated for $1 \mathrm{~h}$ at room temperature while rocking followed by an overlay of $1.5 \%$ SeaPlaque Agarose and modified eagle medium (MEM) supplemented with $10 \%$ fetal bovine serum (FBS, Atlanta Biologicals), $100 \mathrm{U}$ penicillin/mL, and $100 \mu \mathrm{g} / \mathrm{mL}$ streptomycin. After 2 days for MNV-1 and 3 days for MNV-3 and MNV-CR6, cells were stained with $1.5 \%$ SeaKem Agarose in phosphate-buffered saline (PBS) to visualize plaques. Plaques were counted 3-4 h later. In certain experiments, neutral red-labeled virus was used in order to differentiate between inoculum and newly synthesized virus along the intestinal tract ${ }^{31,37}$. When using tissues from mice infected with neutral red virus, samples were exposed to white light for $30 \mathrm{~min}$ prior to titration in order to inactivate input virus.

Histology. Small and large intestinal tissue sections as well as spleen sections were collected from 3-day-old pups infected with mock inoculum or $10^{8} \mathrm{TCID}_{50}$ units MNV-1, MNV-3, or MNV-CR6 at 1 and 2 dpi. Intestinal issues were swiss-rolled and fixed in $10 \%$ buffered formalin for $16 \mathrm{~h}$, transferred to PBS, and then paraffinembedded and sectioned by the University of Florida Molecular Pathology Core. Serial sections of $4-\mu \mathrm{m}$ thickness were cut from each tissue and slides stained with hematoxylin and eosin by the University of Florida Molecular Pathology Core. Tissue sections were imaged using a Nikon Ti-E widefield microscope with a Nikon DS-Fi2 color camera and NIS Elements software at the University of Florida Cell and Tissue Analysis Core. Histology slides were scored blindly by an animal veterinarian based on an established scoring system ${ }^{38}$. Three categories-inflammatory cell infiltrate, epithelial changes, and mucosal architecture-were scored for two criteria each: Inflammatory cell infiltrate was scored for severity $(0=$ none; $1=$ minimal; $2=$ mild with scattered polymorphonuclear neutrophils; $3=$ moderate $)$ and extent $(0=$ none; $1=$ mucosal; $2=$ mucosal and submucosal). Epithelial changes were scored for crypt hyperplasia $(0=2-3 \mathrm{~mm}$ crypt height at $10 \mathrm{X} ; 1=3-4 \mathrm{~mm}$ crypt height; $2=4-5 \mathrm{~mm}$ crypt height; and $3=\geq 5 \mathrm{~mm}$ crypt height) and reduction in goblet cells $(0=$ no reduction; $1=$ minimal reduction). Mucosal architecture was scored for vacuolated epithelium $(0=$ none; $1=$ present at villous tip; $2=$ present and disorganized, seen at the base) and villous tip swelling ( $0=$ none; $1=$ lacteal separation with cellular material in the center; 2 = lacteal dilation). Data are presented as the total pathology score for all criteria per mouse averaged per group (Fig. 3b, c) and separated by category (Supplementary Fig. 2).

RNAscope ISH. RNAscope ISH assays were performed using the RNAscope 2.5 HD Assay-RED kit according to manufacturer's instructions (Advanced Cell Diagnostics, Newark, CA). Formalin-fixed, paraffin-embedded serial sections of intestinal swiss rolls from mock- and MNV-1-infected pups were deparaffinized by heating at $60^{\circ} \mathrm{C}$ for $30 \mathrm{~min}$ followed by xylene treatment and dehydration. Sections were hybridized with custom-designed probes targeting positive-sense or negativesense MNV-1 RNA for $2 \mathrm{~h}$ at $40^{\circ} \mathrm{C}$ prior to probe amplification and detection ${ }^{8}$. Sections were counterstained with $50 \%$ hematoxylin to visualize tissue morphology and imaged using a Nikon DS-Fi2 color camera and NIS Elements software at the University of Florida Cell and Tissue Analysis Core. Sections were scored based on amount of virus present in the GALT, intestinal lamina propria, and intestinal epithelial cells on a scale from 0 to $3(0=0-1$ dots, $1=$ rare staining but at least 2 dots, $2=$ consistent, distinguishable dots throughout the section, $3=$ intense staining with overlapping dots). Positive (PPIB) and negative (DapB) control probes were stained in parallel for all experiments, and six mock mice were stained.

Statistical analyses. All data were analyzed with GraphPad Prism software. Error bars denote standard errors of mean in all figures and $P$ values were determined using one- or two-way ANOVA with corrections for multiple comparisons. In the case of survival curves and incidence of diarrhea, statistical significance was determined using a Mantel-Cox test.

Reporting summary. Further information on research design is available in the Nature Research Reporting Summary linked to this article.

\section{Data availability}

All data that support the findings of this study are included in this paper, the Supplementary information, the Source data file, and/or available from the corresponding author on request. The source data underlying Fig. 1a, b, d, e, Fig. 2a-f, Fig. 3b, c, Fig. 4a, b, Fig. 5b, e, Fig. 6a-c, Supplementary Fig. 1a-e, and Supplementary Fig. $2 \mathrm{a}-\mathrm{c}$ are provided as a source data file.

Received: 24 October 2019; Accepted: 25 May 2020;

Published online: 11 June 2020 


\section{References}

1. Koo, H. L. et al. Noroviruses: the most common pediatric viral enteric pathogen at a large university hospital after introduction of rotavirus vaccination. J. Pediatr. Infect. Dis. Soc. 2, 57-60 (2013).

2. Patel, M. M. Systematic literature review of role of noroviruses in sporadic gastroenteritis. Emerg. Infect. Dis. 14, 1224-1231 (2008).

3. Ahmed, S. M. et al. Global prevalence of norovirus in cases of gastroenteritis: a systematic review and meta-analysis. Lancet Infect. Dis. 14, 725-730 (2014).

4. Cheetham, S. et al. Pathogenesis of a genogroup II human norovirus in gnotobiotic pigs. J. Virol. 80, 10372-10381 (2006).

5. Seo, D. J. et al. Experimental miniature piglet model for the infection of human norovirus GII. J. Med. Virol. 90, 655-662 (2018).

6. Souza, M., Azevedo, M. S. P., Jung, K., Cheetham, S. \& Saif, L. J. Pathogenesis and immune responses in gnotobiotic calves after infection with the genogroup II.4-HS66 strain of human norovirus. J. Virol. 82, 1777-1786 (2008)

7. Karst, S. M., Wobus, C. E., Lay, M., Davidson, J. \& Virgin, H. W. STAT1dependent innate immunity to a norwalk-like virus. Science 299, 1575-1578 (2003).

8. Grau, K. R. et al. The major targets of acute norovirus infection are immune cells in the gut-associated lymphoid tissue. Nat. Microbiol. 2, 1586 (2017).

9. Hsu, C. C., Riley, L. K., Wills, H. M. \& Livingston, R. S. Persistent infection with and serologic crossreactivity of three novel murine noroviruses. Comp. Med. 56, 247-251 (2006)

10. Arias, A., Bailey, D., Chaudhry, Y. \& Goodfellow, I. G. Development of a reverse genetics system for murine norovirus 3; long-term persistence occurs in the caecum and colon. J. Gen. Virol. 93, 1432-1441 (2012).

11. Thackray, L. B. et al. Murine noroviruses comprising a single genogroup exhibit biological diversity despite limited sequence divergence. J. Virol. 81 , 10460-10473 (2007)

12. Wilen, C. B. et al. Tropism for tuft cells determines immune promotion of norovirus pathogenesis. Science 360, 204-208 (2018).

13. Mumphrey, S. M. et al. Murine norovirus 1 infection is associated with histopathological changes in immunocompetent hosts, but clinical disease is prevented by STAT1-dependent interferon responses. J. Virol. 81, 3251-3263 (2007).

14. Kahan, S. M. et al. Comparative murine norovirus studies reveal a lack of correlation between intestinal virus titers and enteric pathology. Virology 421, 202-210 (2011).

15. Zhu, S. et al. Regulation of norovirus virulence by the VP1 protruding domain correlates with B cell infection efficiency. J. Virol. 90, 2858-2867 (2016).

16. Rocha-Pereira, J., Kolawole, A. O., Verbeken, E., Wobus, C. E. \& Neyts, J. Post-exposure antiviral treatment of norovirus infections effectively protects against diarrhea and reduces virus shedding in the stool in a mortality mouse model. Antivir. Res. 132, 76-84 (2016).

17. Strong, D. W., Thackray, L. B., Smith, T. J. \& Virgin, H. W. Protruding domain of capsid protein is necessary and sufficient to determine murine norovirus replication and pathogenesis in vivo. J. Virol. 86, 2950-2958 (2012).

18. Feng, N., Franco, M. A. \& Greenberg, H. B. Murine model of rotavirus infection. Adv. Exp. Med. Biol. 412, 233-240 (1997).

19. Ramig, R. F. The effects of host age, virus dose, and virus strain on heterologous rotavirus infection of suckling mice. Microb. Pathog. 4, 189-202 (1988).

20. Du, J. et al. Detailed analysis of BALB/c mice challenged with wild type rotavirus EDIM provide an alternative for infection model of rotavirus. Virus Res. 228, 134-140 (2017).

21. Cannon, J. L., Lopman, B. A., Payne, D. C. \& Vinjé, J. Birth cohort studies assessing norovirus infection and immunity in young children: a review. Clin. Infect. Dis. 69, 357-365 (2019).

22. Menon, V. K. et al. Norovirus gastroenteritis in a birth cohort in southern India. PLoS ONE 11, e0157007 (2016).

23. Rouhani, $S$. et al. Norovirus infection and acquired immunity in 8 countries: results from the MAL-ED study. Clin. Infect. Dis. 62, 1210-1217 (2016).

24. Agus, S. G., Dolin, R., Wyatt, R. G., Tousimis, A. J. \& Northrup, R. S. Acute infectious nonbacterial gastroenteritis: intestinal histopathology. Histologic and enzymatic alterations during illness produced by the Norwalk agent in man. Ann. Intern. Med. 79, 18-25 (1973).

25. Schreiber, D. S., Blacklow, N. R. \& Trier, J. S. The small intestinal lesion induced by Hawaii agent acute infectious nonbacterial gastroenteritis. J. Infect. Dis. 129, 705-708 (1974)

26. Parrino, T. A., Schreiber, D. S., Trier, J. S., Kapikian, A. Z. \& Blacklow, N. R. Clinical immunity in acute gastroenteritis caused by Norwalk agent. N. Engl. J. Med. 297, 86-89 (1977).

27. Schreiber, D. S., Blacklow, N. R. \& Trier, J. S. The mucosal lesion of the proximal small intestine in acute infectious nonbacterial gastroenteritis. $N$. Engl. J. Med. 288, 1318-1323 (1973).
28. Dolin, R., Levy, A. G., Wyatt, R. G., Thornhill, T. S. \& Gardner, J. D. Viral gastroenteritis induced by the Hawaii agent. Jejunal histopathology and serologic response. Am. J. Med. 59, 761-768 (1975).

29. Troeger, $H$. et al. Structural and functional changes of the duodenum in human norovirus infection. Gut 58, 1070-1077 (2009).

30. Seamons, A. et al. Obstructive lymphangitis precedes colitis in murine norovirus-infected Statl-deficient mice. Am. J. Pathol. 188, 1536-1554 (2018)

31. González-Hernández, M. B., Perry, J. W. \& Wobus, C. E. Neutral red assay for murine norovirus replication and detection in a mouse. Bio-Protoc. 3, e415 (2013).

32. Perry, J. W. \& Wobus, C. E. Endocytosis of murine norovirus 1 into murine macrophages is dependent on dynamin II and cholesterol. J. Virol. 84, 6163-6176 (2010)

33. Ball, J. M., Tian, P., Zeng, C. Q., Morris, A. P. \& Estes, M. K. Age-dependent diarrhea induced by a rotaviral nonstructural glycoprotein. Science 272, 101-104 (1996)

34. Lundgren, $\mathrm{O}$. et al. Role of the enteric nervous system in the fluid and electrolyte secretion of rotavirus diarrhea. Science 287, 491-495 (2000).

35. Istrate, C., Hagbom, M., Vikström, E., Magnusson, K.-E. \& Svensson, L. Rotavirus infection increases intestinal motility but not permeability at the onset of diarrhea. J. Virol. 88, 3161-3169 (2014).

36. Zhu, S. et al. Identification of immune and viral correlates of norovirus protective immunity through comparative study of intra-cluster norovirus strains. PLoS Pathog. 9, e1003592 (2013).

37. Gonzalez-Hernandez, M. B. et al. Efficient norovirus and reovirus replication in the mouse intestine requires microfold (M) cells. J. Virol. 88, 6934-6943 (2014).

38. Erben, U. et al. A guide to histomorphological evaluation of intestinal inflammation in mouse models. Int. J. Clin. Exp. Pathol. 7, 4557-4576 (2014)

\section{Acknowledgements}

S.M.K. was funded by NIH R01AI116892, NIH R01AI081921, and NIH R01AI141478. A N.R. was supported by NIH 5T32AI007110. E.W.H. was supported by NIH T90DE021990. C.E.W. was funded by NIH R01AI141478 and the Biological Sciences Scholars Program at the University of Michigan.

\section{Author contributions}

A.N.R., E.W.H., and S.M.K. designed the study and analyzed results. A.N.R., E.W.H., S.G., and C.M. performed mouse infections, harvests, and plaque assays. E.K. and J.C.S performed plaque assays. L.B.E. scored histology slides in a blinded fashion. A.N.R., E.W.H., and S.M.K. prepared the paper. C.E.W. and N.A-B. edited the paper.

\section{Competing interests}

The authors declare no competing interests.

\section{Additional information}

Supplementary information is available for this paper at https://doi.org/10.1038/s41467 020-16798-1.

Correspondence and requests for materials should be addressed to S.M.K.

Peer review information Nature Communications thanks the anonymous reviewers for their contribution to the peer review of this work

Reprints and permission information is available at http://www.nature.com/reprints

Publisher's note Springer Nature remains neutral with regard to jurisdictional claims in published maps and institutional affiliations.

Open Access This article is licensed under a Creative Commons Attribution 4.0 International License, which permits use, sharing, adaptation, distribution and reproduction in any medium or format, as long as you give appropriate credit to the original author(s) and the source, provide a link to the Creative Commons license, and indicate if changes were made. The images or other third party material in this article are included in the article's Creative Commons license, unless indicated otherwise in a credit line to the material. If material is not included in the article's Creative Commons license and your intended use is not permitted by statutory regulation or exceeds the permitted use, you will need to obtain permission directly from the copyright holder. To view a copy of this license, visit http://creativecommons.org/ licenses/by/4.0/.

(c) The Author(s) 2020 